\title{
Investigating the relationship between background luminance and self-reported valence of auditory stimuli
}

\author{
I.K. Wardhani ${ }^{*}$, B.H. Janssen, C.N. Boehler \\ Department of Experimental Psychology, Ghent University, Belgium
}

\section{A R T I C L E I N F O}

\section{Keywords:}

Luminance

Auditory perception

IADS-E

Valence rating

Pupil size

Pupillometry

\begin{abstract}
A B S T R A C T
The present study investigated the effect of background luminance on the self-reported valence ratings of auditory stimuli, as suggested by some earlier work. A secondary aim was to better characterise the effect of auditory valence on pupillary responses, on which the literature is inconsistent. Participants were randomly presented with sounds of different valence categories (negative, neutral, and positive) obtained from the IADS-E database. At the same time, the background luminance of the computer screen (in blue hue) was manipulated across three levels (i.e., low, medium, and high), with pupillometry confirming the expected strong effect of luminance on pupil size. Participants were asked to rate the valence of the presented sound under these different luminance levels. On a behavioural level, we found evidence for an effect of background luminance on the selfreported valence rating, with generally more positive ratings as background luminance increased. Turning to valence effects on pupil size, irrespective of background luminance, interestingly, we observed that pupils were smallest in the positive valence and the largest in negative valence condition, with neutral valence in between. In sum, the present findings provide evidence concerning a relationship between luminance perception (and hence pupil size) and self-reported valence of auditory stimuli, indicating a possible cross-modal interaction of auditory valence processing with completely task-irrelevant visual background luminance. We furthermore discuss the potential for future applications of the current findings in the clinical field.
\end{abstract}

\section{Introduction}

In the realm of human emotion, the appropriate emotional evaluation of diverse and multisensory environmental stimuli is important, not least because many of our decisions and (emotional) experiences are made based on the integration of these stimuli. In research settings, emotion is often measured on valence, arousal, and dominance dimensions (Bradley \& Lang, 1994). Valence describes whether a stimulus is positive (pleasant), neutral, and negative (aversive). Arousal describes the degree of stimulation or intensity of that stimulus. Dominance refers to how influential, controlling, or important that stimulus is for an individual. For instance, repetitive tapping sounds can be perceived as unpleasant (i.e., the valence) by two different individuals. However, the dominance and/or arousal can be perceived as very high by one but low by another individual, depending on personal and situational factors.

Emotion studies largely use visual stimuli, ranging from simple colours to pictures of facial expressions, in order to evoke emotions (e.g., Lang et al., 1993; Sánchez-Navarro et al., 2006; Wilms \& Oberfeld, 2018). One important example of studies in this field comes from Lang et al. (1993), who used coloured photographs as stimuli and linked the participants' valence judgements to physiological features such as activations in facial muscles, heart rate, and skin conductance. While for a long time, the bulk of emotion research has employed visual stimuli, the investigation of auditory stimuli to study emotional evaluation and experience has received increasing attention (Bradley \& Lang, 2000; Redondo et al., 2008; Soares et al., 2013; Yang et al., 2018). Parallel to visual stimuli, it has been shown that auditory stimuli can also be indexed by valence, arousal, and dominance in similar ways as visual stimuli (Bradley \& Lang, 2000). Specifically, Bradley and Lang (2000) demonstrated that unpleasant sounds evoke more facial muscle activity beneath the eye and a larger startle reflex. Listening to emotional sounds, as compared to neutral sounds, also elicits greater skinconductance responses. Furthermore, highly arousing sounds (both pleasant and unpleasant) are later better recalled than low or nonarousing sounds. These detailed findings have led to an increased interest in the emotional effects of sounds, and in the use of sound samples as standardised in the International Affective Digitised Sounds (IADS) database (Bradley \& Lang, 1999, 2007; Yang et al., 2018).

\footnotetext{
* Corresponding author at: Department of Experimental Psychology, Faculty of Psychological and Educational Sciences, Henri Dunantlaan 2, Ghent 9000, Belgium.

E-mail address: intankusuma.wardhani@ugent.be (I.K. Wardhani).
} 
In the visual domain, a few studies have also started using more abstract stimuli, and demonstrated similar effects as studies using pictures and sounds as stimuli. For instance, Wilms and Oberfeld (2018) looked at the effects of hue, saturation, and luminance on the valence and arousal dimensions of emotion. Alongside three achromatic colours as a baseline, they presented 27 chromatic colours via an LED display, on which participants were instructed to fixate their gaze for $30 \mathrm{~s}$. Afterwards, while the colour was still on display, participants were asked to rate their emotional states on the valence and arousal scales of the SelfAssessment Manikin (SAM; Bradley \& Lang, 1994). At the same time, participants' skin conductance response and heart rate were recorded. The results indicated that highly saturated and luminant colours, especially blue, were associated with the most positive valence rating. Moreover, regardless of the colours, high saturation and luminance were associated with a stronger skin conductance response and heart rate acceleration. These results (Wilms \& Oberfeld, 2018) show that even abstract stimuli like colours can be explicitly graded on valence and arousal, and importantly also modulate one's emotional state as measured with more implicit, physiological measures.

Nevertheless, in real life the strict distinction of visual and other stimuli is somewhat artificial because stimuli come in a multisensory fashion. Yet, although some research has been devoted to multisensory integration in general (Noesselt et al., 2010; Spence, 2007; Stein et al., 1996; Stein \& Stanford, 2008; Talsma et al., 2010; Thompson et al., 1958), only relatively little research has been devoted to investigating possible interactions between multisensory inputs of affective stimuli (but see also Nakakoga et al., 2020). Importantly, multisensory inputs here can refer to a given real-life object (e.g., a snake that is hissing), but can also refer to more general interactions (e.g., a certain sound under bright or dark lighting conditions). A particularly interesting study that used both visual and auditory stimuli, which furthermore extended it to the realm of pupillometry, comes from Cherng et al. (2020). They used the combination of varied luminance levels, emotional auditory stimuli, and a pro-/anti-saccade (cognitive) task specifically for examining the different contributions of sympathetic activation and parasympathetic inhibition on pupillary responses. They found that pupil dilation was larger with the dark than bright background when evoked by emotional stimuli (i.e., sympathetic activation) and vice versa for the cognitive task (i.e., parasympathetic inhibition). This finding supported the conjecture that pupillary responses evoked by emotion and arousal (i.e., psychosensory pupil response) relied on the sympathetic control pathway (Mathôt, 2020; McDougal \& Gamlin, 2008). However, it did not illuminate the relationship between the visual and auditory stimuli because they did not directly measure whether the different background luminance modulates emotional experiences or, more specifically, valence response.

\subsection{The present study}

Given the scarce work directly addressing the interaction of background luminance and the processing of valenced auditory stimuli, our primary goal in the present study was to investigate the effects of multimodal sensory inputs (i.e., visual and auditory) on emotional appraisal as measured by behavioural ratings. To this end, we incorporated elements of Cherng et al.'s (2020) findings with those of Wilms and Oberfeld (2018) and investigated whether task-irrelevant luminance (in varied levels) would modulate participants' explicit appraisal of affective auditory stimuli. As demonstrated by Wilms and Oberfeld (2018), a highly luminant blue hue was rated more positively, suggesting a positive influence on participants' emotional state. However, Wilms and Oberfeld (2018) presented the changes in luminance alone rather than with another stimulus. In our current study, we presented different sounds on varied background luminance. Therefore, our first research question here was whether background luminance would modulate the valence judgements of auditory emotional stimuli. Based on the work by Wilms and Oberfeld (2018), we expected a general positive shift of valence ratings for higher background luminance, i.e., a main effect of background luminance on valence ratings.

As our secondary goal, we were interested in investigating how valence of sounds per se (and independent of background luminance) would be reflected in pupillary responses, not least because previous findings have been equivocal. In previous years, many studies have indicated that pupils respond to arousing or intense stimuli regardless of the valence (Hess \& Polt, 1960, 1964; Kahneman \& Beatty, 1966; Mathôt, 2018). A classic study by Hess (1965) showed that pupils constricted in response to repeated negative stimuli but dilated when exposed to repeated positive visual stimuli (see also de Winter et al., 2021). In contrast to that finding, Partala et al. (2000) showed the opposite pattern of pupil dilations during the presentation of emotional stimuli. In their Experiment 2, they attempted to equalise the type of emotional auditory stimuli by selecting relatively similar contents in each valence category. They found that negative stimuli evoked the largest pupil dilation and no difference in pupil size evoked by the positive and neutral stimuli. Similarly, Kawai et al. (2013) found significantly larger pupil dilation for negative stimuli relative to positive stimuli. However, many other studies seem to contradict the notion that pupils respond differently to positive vs. negative stimuli (Bradley et al., 2008; Janisse, 1974; Oliva \& Anikin, 2018; Partala et al., 2000; Partala \& Surakka, 2003). These studies show that pupils dilate similarly during the presentation of negative and positive stimuli, and that pupil size is in turn smaller in response to neutral stimuli. In line with this, also in Cherng et al.'s (2020) study, pupil dilations in the negative and positive valence conditions were both larger than in the neutral valence condition. In summary, there is therefore still a need for further data to better understand the relationship between valence and pupil size.

Our second research question thus aimed to explore further the relationship between the valence of sounds and pupil size in a wellcontrolled experiment with a relatively large sample size. We hypothesised that the valence of the presented sound samples would be reflected differently in pupil size. Specifically, we hypothesised that pupil size in the negative sound conditions would be larger than pupil size in the neutral sound condition. We did not have an expected trend of pupil size in the positive sound condition relative to the negative and neutral sound conditions because the literature showed divergent results.

Of note, when considering both research goals, it is important to realise that we therefore were interested in separate main effects of luminance for behaviour and valence for pupil responses, both of which are rather trivial in the other data modality (i.e., different valence ratings for the different sound categories are very much expected, as are differential pupil responses to different background luminance levels). While the interaction of background luminance and valence would be interpretable in both modalities, and are explored here, no such interactions were in fact expected.

\section{Method}

\subsection{Participants}

Forty student volunteers ( $n$ women $=33, M_{\text {age }}=19.32$ years, $S D=$ 3.48 , age range $=18-40$ years) at Ghent University participated in the present study. A post-hoc power analysis using G*Power (Faul et al., 2007) revealed that we reached at least $97.74 \%$ power to detect an effect with our sample size. Before starting the experiment, participants read and signed an informed consent form. Participation took $1 \mathrm{~h}$ and was compensated with a course credit. All participants reported normal hearing on both ears and normal/corrected-to-normal vision. Debriefing was sent via email by the experimenter to each participant after the experiment was concluded. The current research was carried out in accordance with the ethical rules for human participants stipulated in the Declaration of Helsinki. 


\subsection{Design and materials}

We obtained the permission to use the auditory stimuli from the IADS-E database (Yang et al., 2018). We selected a set of auditory stimuli to include stimuli only with the arousal and dominance values in the middle range, such that variations in arousal and dominance levels were reduced (Appendix A). We furthermore selected auditory stimuli that were not extremely negative or positive because highly negative stimuli were, on average, rated as more arousing than the highly positive stimuli, rendering the arousal matching more difficult (Table 1). To categorise the valence of the sounds, we arbitrarily selected stimuli with the valence mean value below 4 as Negative, between 4 and 6 as Neutral, and above 6 as Positive sounds. The selection of the auditory stimuli resulted in 35 stimuli for each Sound category. While actively trying to match arousal and dominance across the different valence categories, there is a general relationship that could not fully be avoided. While small, the residual relationship between valence and arousal is of theoretical concern, because arousal is an important parameter influencing pupil size (Bradley et al., 2008; Mathôt, 2018). To preview, in order to control for this, we ran an additional analysis confirming our general result pattern using only a subset of the stimuli that were then perfectly matched for arousal (see Results).

To manipulate the Luminance levels, we used the hue-saturationvalue (HSV) system and changed the $V$ value while keeping the $H$ and $S$ values constant. In the experiment, we implemented Low (HSV: $210^{\circ}$, $88 \%, 60 \%$ ), Medium (HSV; $210^{\circ}, 88 \%, 70 \%$ ), and High (HSV: $210^{\circ}$, $88 \%, 80 \%$ ) luminance. These values correspond to a bluish hue and were selected because it has been shown to have positive effects on valence as luminance increases (Wilms \& Oberfeld, 2018).

The same auditory stimuli per valence category were used in each luminance level ( 35 sounds $\times 3$ Sound categories $\times 3$ Luminance levels). In total, every participant performed 315 fully randomised trials. Each trial started with a black fixation cross on a blue screen of varying luminance for $1 \mathrm{~s}$ (Fig. 1). Next, participants fixated their gaze on a fixation dot on the screen while listening to a sound for $4 \mathrm{~s}$. After that, participants rated the valence of the sound on a continuous rating scale (ranging from "Very negative" to "Very positive") by using a computer mouse and with no time limit. Finally, there was an intertrial interval of $1 \mathrm{~s}$. Each trial had the same luminance level throughout all phases.

We used OpenSesame (Mathôt et al., 2012) to create and execute the experiment. Visual stimuli were presented on an LCD screen (BenQ XL2420TE, $24^{\prime \prime}$ ) with a resolution of $1920 \times 1080 \mathrm{px}$ and a refresh rate of $60 \mathrm{~Hz}$. Auditory stimuli were delivered to both ears at a constant volume that was comfortable for all participants via a pair of headphones (Sennheiser HD 280 Pro). Pupil size was recorded during the experimental task by an EyeLink 1000+ desktop-mount eyetracker (SRResearch, Ontario, Canada) with a sampling rate of $500 \mathrm{~Hz}$.

\subsection{Procedure}

Participants used a chin rest to maintain a stable distance of $54 \mathrm{~cm}$

Table 1

Mean values of the sound samples.

\begin{tabular}{|c|c|c|c|}
\hline \multirow[t]{2}{*}{ Category } & \multicolumn{3}{|l|}{ Dimension } \\
\hline & Arousal & Dominance & Valence \\
\hline Negative & $\begin{array}{l}5.33(0.44 \\
4.41-5.96)\end{array}$ & $\begin{array}{l}4.60(0.37 \\
4.00-5.50)\end{array}$ & $\begin{array}{l}3.49(0.51 \\
1.50-3.92)\end{array}$ \\
\hline Neutral & $\begin{array}{l}5.07(0.43 \\
4.00-6.00)\end{array}$ & $\begin{array}{l}5.12(0.47 \\
4.18-5.95)\end{array}$ & $\begin{array}{l}4.98(0.12 \\
4.82-5.18)\end{array}$ \\
\hline Positive & $\begin{array}{l}4.91(0.37 \\
4.00-6.00)\end{array}$ & $\begin{array}{l}5.46(0.36 \\
4.36-6.00)\end{array}$ & $\begin{array}{l}6.70(0.47, \\
6.04-7.86)\end{array}$ \\
\hline
\end{tabular}

Note. The table shows the mean (standard deviation, range) values of the sound samples obtained from IADS-E in each dimension and per sound category used in the current study. between the cornea and the eye-tracker's lens. The illumination in the room was at a constant ambient-room level and the experimenter was present in the room for all participants. An opaque divider was placed between the participant and the experimenter to avoid distraction.

The experimenter started a nine-point eye-movement calibration and validation once the participant was seated. Afterwards, participants read the instruction on the computer screen. Participants were asked to rate their emotional response towards the presented sound stimuli on an analogue scale using the computer mouse. It was emphasised that there was no correct or incorrect response. The experiment started with one practice session to familiarise the participants with the use of the rating scale. Afterwards, they were informed that the real experiment would start.

After every nine trials, participants had a break of $10 \mathrm{~s}$ to avoid eye fatigue. The short break was indicated by a high beep and a written announcement on the computer screen ("Short break of $10 \mathrm{~s}$ "). After $5 \mathrm{~s}$, participants heard a low beep to indicate that the break was half passed. After $7 \mathrm{~s}$, a high beep was presented again with a written announcement, "Short break ends soon". After 157 trials, there was a break of $2 \mathrm{~min}$. The long break was indicated by a high beep and a written announcement on the computer screen ("Long break of 2 minutes"). Three seconds before the long break ended, "Long break ends soon" was announced on the computer screen. There was a high beep to announce the ending of the long break. Pupil size was not recorded during breaks.

\section{Data analysis}

We used Python 3.7 on Spyder 5.0.1 (https://www.spyder-ide.org/) to process the behavioural and pupillometric data, after which was exported to JASP (JASP Team, 2020) for statistical analysis. In all analyses, we performed repeated-measures ANOVAs with the GreenhouseGeisser sphericity correction. Post-hoc multiple comparisons were performed for significant main effects. To report the multiple-comparison $p$ values, we used the Holm correction and did not pool the error terms of the repeated-measures factors. Our dataset and an example task are available on our Open Science Framework page. Our preprocessing analysis scripts are available upon request.

\subsection{Valence rating data}

Participants' ratings of the sound samples were collected in a continuous scale ranging from 0 (very negative) to 100 (very positive). During the analysis, we converted the scale such that it ranged from -100 (very negative) to 100 (very positive). The aggregated mean values were taken and analysed using a 3 (Sound: negative, neutral, positive) $\times 3$ (Luminance: low, medium, high) repeated-measures ANOVA. As post-hoc tests following significant effects, we performed Holm-corrected one-tailed $t$-tests using the pairwise_ttests function of the Python's pingouin package (Vallat, 2018). These one-tailed tests were performed because of the expected direction in our first hypothesis (see The present study).

\subsection{Pupillometric data}

For the analysis related to the different background luminance onset, we used an epoch of interest to $0-5 \mathrm{~s}$ time-locked to the onset of the fixation stimulus and simultaneous change in background luminance (see Figs. 1 and 3). To characterise differences in the actual pupil size during this epoch, pupillary light reflexes to the different background luminance were analysed using the absolute values without baseline correction (similar starting values could be assumed, and were also observed, based on the random trial sequence). For analyses related to the sound stimuli, we time-locked the epoch of interest to the onset of the sound stimuli, and hence $1 \mathrm{~s}$ after the change in background luminance, considering a four-second time-period (i.e., $1-5 \mathrm{~s}$ with regard to the background luminance change; see Figs. 1 and 4). This time, we 


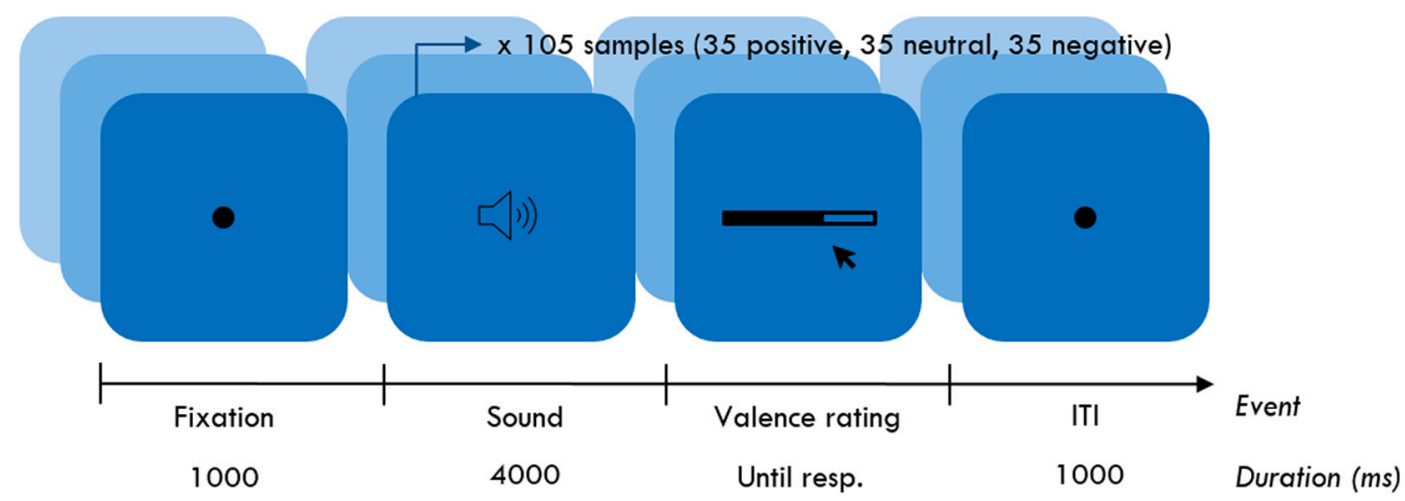

Fig. 1. Trial sequence of the experiment.

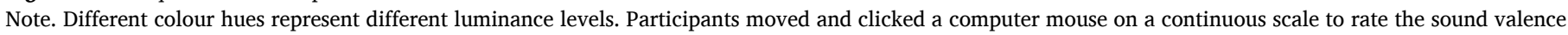

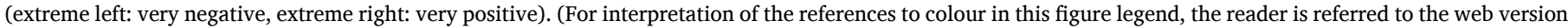
of this article.)

performed a baseline correction using a subtractive method, in which values from the baseline period were subtracted from every data point thereafter (see Mathôt et al., 2018). We used the median values of the first $400 \mathrm{~ms}$ since sound onset (1-1.4 s with regard to the background luminance change) as the baseline period. We chose this baseline period because there was minimal variance due to luminance differences, while still clearly preceding the typical latency of pupil responses to cognitive processing (Laeng et al., 2012; Mathôt, 2018; van der Wel \& van Steenbergen, 2018). Missing data due to eye blinks in that epoch were reconstructed using a cubic spline interpolation (Mathôt, 2013). No further pre-processing for eye-position artefacts was performed.

We performed a one-way analysis (Luminance: low, medium, high) on the absolute pupil size to investigate the effect of background luminance and a 3 (Sound: negative, neutral, positive) $\times 3$ (Luminance: low, medium, high) analysis on the baseline-corrected pupil size data timelocked to auditory stimulus onset. First, we inspected the effect of only background luminance to demonstrate that luminance differences had the expected large effect on pupil size. To this end, we compared the median of absolute pupil size from the luminance (i.e., at fixation) onset to the sound offset (Fig. 1) at each Luminance level averaging over all Sound levels. Second, we investigated how valence was reflected in pupil size. To do that, we aggregated the median values of baselinecorrected pupil size from the epoch of interest every $1.2 \mathrm{~s}$ excluding the baseline period, resulting in three time-windows (i.e., 0.4-1.6 s, 1.6-2.8 s, and 2.8-4 s). Here, we analysed the baseline-corrected pupil size to attenuate (albeit not fully) the strong effect of background luminance on pupil size (which was already characterised by the previous analysis). We used a time-resolved analysis because pupil-size differences due to valence can occur at different time-points and tend to be more pronounced during peak dilations (Partala et al., 2000; van Rij et al., 2019). In time-resolved analysis, we corrected the $p$-values using the Benjamini-Hochberg false-discovery-rate correction (FDR correction) method (Benjamini \& Hochberg, 1995). ${ }^{1}$ The FDR correction was carried out using the multicomp and pairwise_ttests functions of the

\footnotetext{
${ }^{1}$ While also reporting the main effect of luminance after baseline correction, which strongly attenuates the size of the pupillary light reflex, we note that a remaining main effect even after this procedure is most likely still related to background luminance. In fact, since differences in pupil size based on luminance condition were maximal briefly before the presentation of the emotional sounds and then decreased, the baseline correction turned around what was expected and observed for absolute pupil size, with largest baseline-corrected values for the highest luminance condition. While effects of luminance in this later time-range could theoretically represent a main effect of luminance condition on the processing of emotional stimuli (akin to such a main effect in the behavioural data), this is impossible to de-confound in the present design.
}

Python's pingouin package (Vallat, 2018). For completeness, we also explored the interaction effect between sound and luminance on baseline-corrected pupil size, although we did not expect such an effect.

\section{Results}

\subsection{Valence ratings}

The main goal of this study was to explore the relationship between background luminance and valence ratings of auditory emotional stimuli. We therefore first analysed the valence ratings that participants gave to the sound stimuli (see Fig. 2). Across all luminance levels, the average valence rating for the negative sounds was $M=-35.06(S D=$ $39.33)$, neutral sounds $M=1.53(S D=43.20)$, and positive sounds $M=$ $39.73(S D=37.20)$. As expected, the statistical results suggested a significant main effect of Sound category $F(1.36,53.055)=383.807, p<$ $.001, \eta_{\mathrm{p}}^{2}=0.908$, indicating that participants indeed correctly rated the valence of the stimuli. More importantly, and in line with our prediction, we found a significant effect for the main effect of Luminance, $F(1.966$, $76.675)=3.136, p=.049, \eta_{p}^{2}=0.074$. Results of the one-tailed posthoc test revealed significant differences between Low-Medium $(t[39]=$ $\left.-2.145, p_{\text {holm }}=.046\right)$ and Low-High $\left(t[39]=-2.243, p_{\text {holm }}=.046\right)$ luminance levels. The difference between Medium-High luminance levels was not significant $\left(t[39]=-0.296, p_{\text {holm }}=.384\right)$. On average, this effect was explained by more positive ratings in the medium and high than in the low background luminance in all valence categories. Hence, the current result provided support to reject the null hypothesis. Concerning a possible interaction between background luminance and emotional sounds, the analysis indicated no interaction effect, $F(3.510$, $136.875)=0.775, p=.528, \eta_{\mathrm{p}}^{2}=0.019$. The absence of the interaction effect suggested that background luminance had no differential effect on valence ratings across sound categories.

\subsection{Pupillary changes}

\subsubsection{Absolute pupil size: effect of luminance on pupillary responses}

We now turned to the secondary research question of how valence affects pupil dilations. Yet, before doing so, we looked at the main effects of background luminance (in order to demonstrate that luminance differences had a large effect on pupil size). To inspect the effect of background luminance, we performed a one-way ANOVA on the median of absolute pupil size since the onset of fixation and the concomitant luminance manipulation until the offset of sound (i.e., not in timeresolved fashion for the entire time period) averaging over all soundvalence levels. The results indicated a significant main effect of background luminance on absolute pupil dilations, $F(1.433,55.904)=$ 

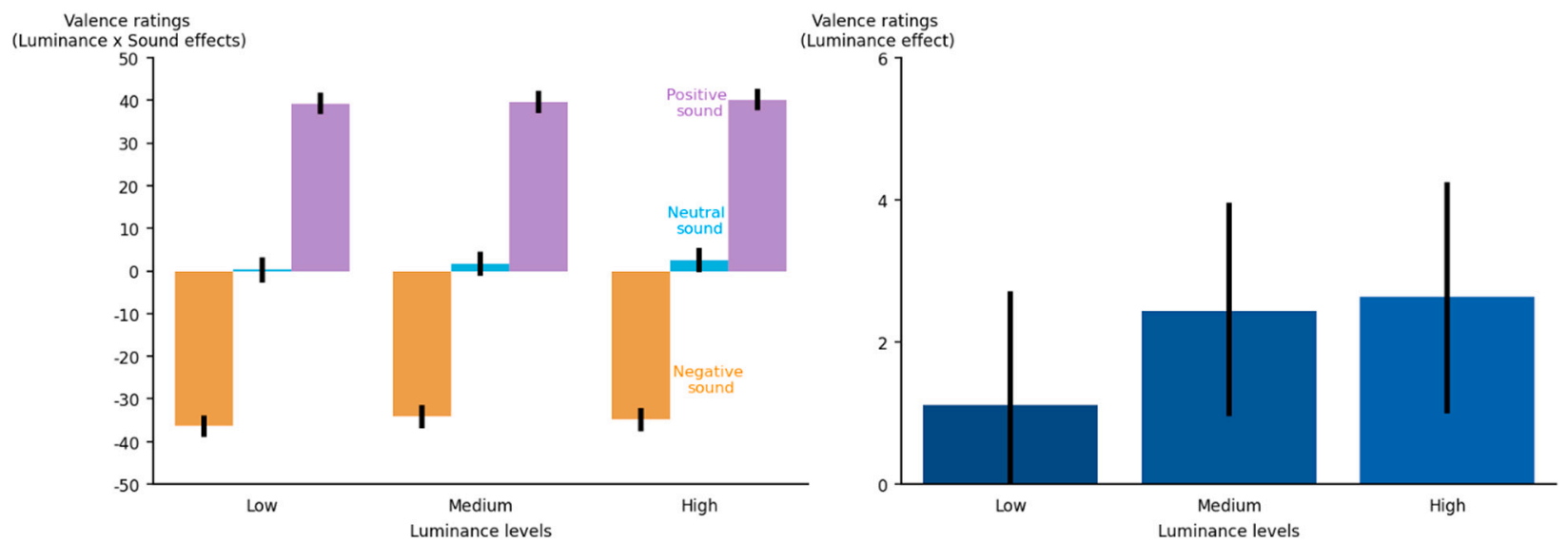

Fig. 2. Valence ratings as a function of background luminance and sound valence.

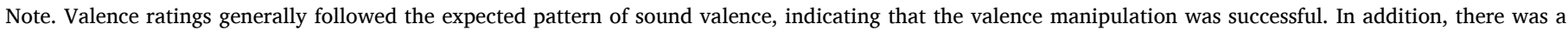

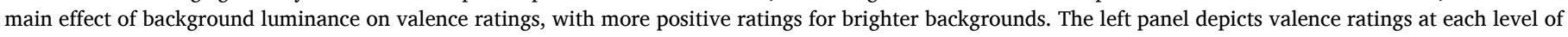

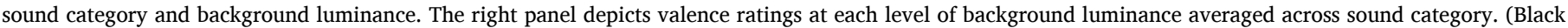
error bars represent a 95\% confidence interval [CI].)

101.9, $p<.001, \eta_{\mathrm{p}}^{2}=0.723$ (Fig. 3). It was evident that pupil size was largest in the Low Luminance $(M=1722, S D=794.8)$ and smallest in the High Luminance $(M=1440, S D=692.6)$ conditions, with the Medium Luminance condition in between the two $(M=1601, S D=774.5)$. Differences were observed in all three post-hoc comparisons: Low vs. Medium $\left(M_{\text {diff }}=121.8, S E=14.76, p_{\text {holm }}<.001\right)$, Low vs. High $\left(M_{\text {diff }}=\right.$ $\left.282.3, S E=25.14, p_{\text {holm }}<.001\right)$, Medium vs. High $\left(M_{\text {diff }}=160.6, S E=\right.$ $\left.18.18, p_{\text {holm }}<.001\right)$. This result demonstrated that the luminance manipulation had the expected strong pupillary responses. Also note that the maximum difference in pupil size was already reached approximately when the sound stimuli were presented but remained visible in the entire time-window (in line with the fact that background luminance remained different across luminance conditions).

\subsubsection{Baseline-corrected pupil size: effect of valence on pupillary responses}

Thereafter, we performed a 3 (Luminance: low, medium, high) $\times 3$ (Sound: negative, neutral, positive) analysis to examine the main effect of sound valence on the baseline-corrected pupil dilations, while also exploring the main effect of luminance and their interaction for completeness. Starting with the main effect of luminance, the results indicated a significant effect in all three time-windows, all $p s<.001$. These results showed that luminance still had a strong effect on pupil size even after the baseline correction. As can be seen in Fig. 5, for example, pupil dilations in the high-luminance condition were the largest and in the low-luminance condition were the smallest, with pupil dilations in the medium-luminance condition in the middle. Of important note, this occurred likely because there was an ongoing nonaffective effect of luminance on pupil size even after the sound onset (e.g., see Fig. 3). Therefore, it would be impossible to dissociate the affective effect from the physical (pupil light reflex) effect of luminance on pupil size.

Turning to the main effect of Sound valence, which was our main interest in this analysis, we compared the baseline-corrected pupil dilations in three time-windows after sound onset $(0.4-1.6 \mathrm{~s}, 1.6-2.8 \mathrm{~s}$, and $2.8-4 \mathrm{~s}$ ). The results after the FDR-correction suggested a significant main effect of Sound valence for the first two time-windows [0.4-1.6 s: $F(1.767,68.897)=12.031, p<.001, \eta_{\mathrm{p}}^{2}=0.235 ; \mathbf{1 . 6 - 2 . 8}$ s: $F(1.890$, 73.697) $\left.=17.409, p<.001, \eta_{\mathrm{p}}^{2}=0.307\right]$ since the onset of sound
Absolute pupil

size (a.u.)

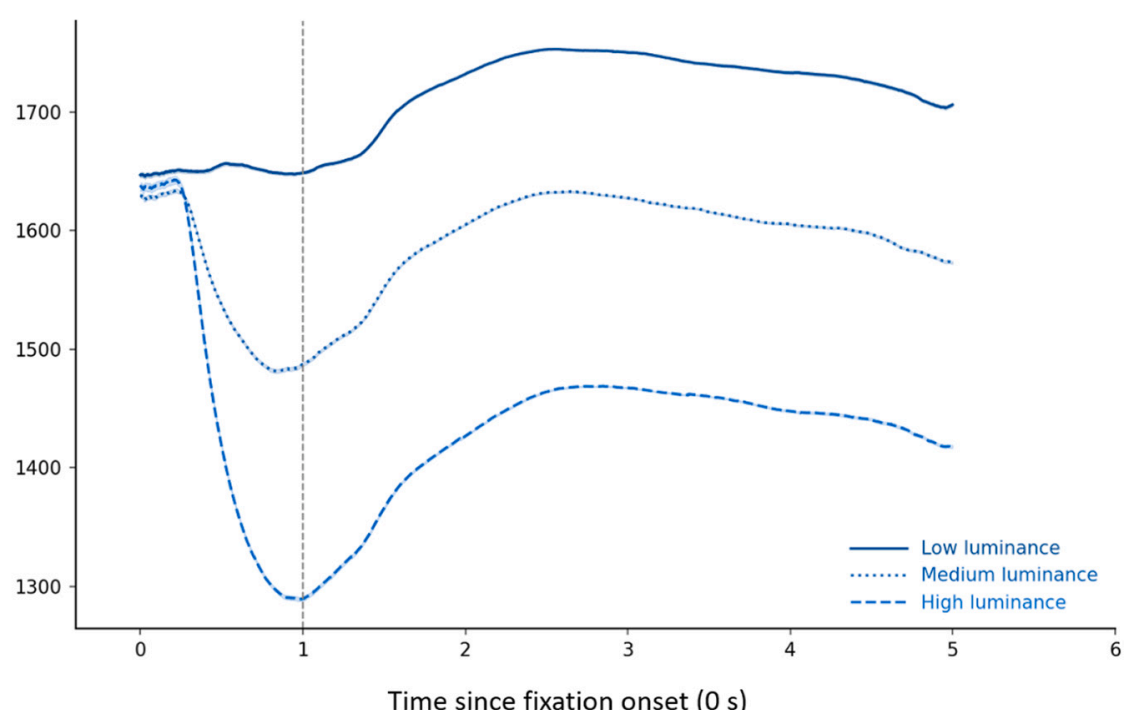

Fig. 3. Pupillary responses to background luminance. Note. Characterising the effect of background luminance on absolute pupil size, averaging over all sound valence levels. The time $0 \mathrm{~s}$ indicates the start of the fixation phase at which background luminance was adjusted according to the respective condition. The vertical dashed line at $1 \mathrm{~s}$ indicates sound onset. As expected, there are large differences in pupil size at each luminance level. (Coloured shades around the pupil trace, although very narrow, represent 95\% CI.) (For interpretation of the references to colour in this figure legend, the reader is referred to the web version of this article.) 
presentation (Fig. 4). The effect was still present but got weaker in the last time window $\left[2.8-4\right.$ s: $F(1.949,75.995)=4.985, p=.015, \eta_{p}^{2}=$ 0.112]. Subsequently, we performed a post-hoc analysis for the three time-windows with significant main effect. In the first time-window, the result revealed that pupil dilation in the Negative condition was larger than pupil dilation in the Positive condition $\left(M_{\text {diff }}=16.865, S E=\right.$ $\left.3.080, p_{\text {holm }}<.001\right)$. Similarly, pupil dilation in the Negative condition was larger than pupil dilation in the Neutral condition $\left(M_{\text {diff }}=9.782, S E\right.$ $\left.=3.168, p_{\text {holm }}=.011\right)$. However, no significant difference was observed between the Neutral and Positive conditions $\left(M_{\mathrm{diff}}=7.083, S E=4.030\right.$, $\left.p_{\text {holm }}=.087\right)$. In the second time window, all comparisons were significant with the largest difference between Negative and Positive conditions $\left(M_{\text {diff }}=31.80, S E=6.037, p_{\text {holm }}<.001\right)$, as in the first timewindow. Pupil dilation in the Negative condition was also larger than in the Neutral condition $\left(M_{\text {diff }}=13.01, S E=5.129, p_{\text {holm }}=.016\right)$ and larger in the Neutral than in the Positive conditions $\left(M_{\text {diff }}=18.79, S E=\right.$ $\left.5.035, p_{\text {holm }}=.002\right)$. In the last time-window, the difference between the Negative and Positive conditions was still significant $\left(M_{\text {diff }}=19.78\right.$, $\left.S E=7.293, p_{\text {holm }}=.046\right)$, as was the difference between the Neutral and Positive conditions $\left(M_{\text {diff }}=16.968, S E=6.385, p_{\text {holm }}=.046\right)$, but not the difference between Negative and Neutral. ${ }^{2}$ These results indicated that valence delivered through sounds was reflected differently in pupil dilations. Moreover, the differences seemed to take place approximately within the first $3 \mathrm{~s}$ after sound onset and especially during a peak dilation.

Finally, we probed the question whether background luminance interacted with sound valence and together modulated the pupil dilations. The resulting interaction effect of Luminance $\times$ Sound was not significant in all three time-windows (Fig. 5), all ps $>.35$.

\section{Discussion}

In the present study, we were primarily interested in the multisensory influences on emotional appraisal as observed through behavioural ratings. Specifically, we investigated whether (task-irrelevant) background luminance would modulate the valence of auditory stimuli. To test this, we presented sound samples obtained from the IADS-E (Yang et al., 2018) in three valence categories (i.e., negative, neutral, positive). We selected only sound samples such that the arousal and dominance dimensions were in the middle range (mean ratings of around 5 for both dimensions). At the same time, we varied the background luminance levels of the computer screen (i.e., low, medium, high) and measured participants' valence ratings of the sound samples and pupillary responses. Our results were in line with our expectation that a brighter background would lead to more positive valence ratings in general. In addition to this main question, we further explored the effect of valence on pupillary responses. Here, we observed largest pupil sizes for negative stimuli, followed by neutral and only then positive stimuli.

\subsection{The effect of background luminance on emotional processing}

First, we hypothesised that sound samples in the high-luminance condition would have more positive valence ratings than those in the low-luminance condition. According to a previous finding (Wilms \& Oberfeld, 2018), higher luminance of a blue hue was associated with a more positive valence rating of the observer's emotional state. In our experiment, we did not test the influence of background luminance

\footnotetext{
${ }^{2}$ Since our full stimulus set still featured a small difference concerning arousal for the different valence categories, we ran an additional analysis in which we dropped a couple of stimuli in order to perfectly match arousal across valence conditions. Specifically, we excluded 8 samples from negative, 5 from neutral, and 8 from positive categories and reanalysed the pupil responses. The results showed qualitatively the same results, hence suggesting that the present results are not due to a systematic difference in arousal levels.
}

directly on the observers' emotional states (in the sense of indicating how they felt and/or how they evaluated the background luminance itself). Rather, we measured the valence ratings of the participants when different sound samples were presented while participants were looking at a screen with different background luminance. Our behavioural results corroborated a strong effect of sound on participants' valence ratings (i.e., that participants perceived the valence of the stimuli as expected). Turning to our main hypothesis that luminance would affect valence ratings, our results supported this prediction. By varying luminance and keeping the hue and saturation at constant levels, our current behavioural findings were in line with those of Wilms and Oberfeld (2018), showing more positive valence ratings in higher luminance.

Emotion studies primarily use either visual or auditory stimulus, but rarely a combination of both. For example, previous studies have used different visual stimuli (e.g., luminance and an image or a colour) and have shown that a more luminant image/colour is often evaluated more positively (Lakens et al., 2013; Suk \& Irtel, 2010). Here, we turned to the relationship between background luminance and the processing of affective sounds. As already indicated, it has been shown that a luminant blue hue has been shown to be associated with a positive emotional appraisal (Suk \& Irtel, 2010; Valdez \& Mehrabian, 1994; Wilms \& Oberfeld, 2018). Here, we showed that this effect extends beyond just the explicit appraisal of that stimulus itself, but also affects the appraisal of auditory stimuli presented simultaneously (Bradley \& Lang, 2000; Lang et al., 1997). In other words, due to the strong visual effect that the observers are experiencing, it may influence the appraisal of other stimulus coming from a different sensory system. On a physiological level, the intrinsically photosensitive retinal ganglion cells (ipRGCs) may be a possible pathway through which luminance affects emotional processing. The colour blue is known to induce a strong response from the ipRGCs (Berson et al., 2002; Gooley et al., 2012; McDougal \& Gamlin, 2008). Some studies have also indicated that the ipRGCs processing is projected to brain regions relevant for emotional processing (Alkozei et al., 2021; Killgore et al., 2020; Vandewalle et al., 2007). For example, Alkozei et al. (2021) suggested that blue light might enhance emotional experience by strengthening the bidirectional information flow between amygdala and left dorsolateral prefrontal cortex. Killgore et al. (2020) also proposed that blue light enhanced emotional experience through the increased neural efficiency, as seen in reduced activation in the neural network for attention-demanding tasks (i.e., Task Positive Network/TPN). From these previous findings, we speculate that an activation of ipRGCs modulate neural processes relevant for the emotional processing of an auditory stimulus as well.

\subsection{The effect of valence on pupillary dilations}

Second, we expected that pupil size in the negative sound condition would be larger than pupil size in the neutral sound condition. However, we did not have an expectation for how pupils would respond to positive sounds relative to negative and neutral sounds. Our second hypothesis was driven by the heterogeneous results in the literature (Bradley et al., 2008; Cherng et al., 2020; Janisse, 1974; Kawai et al., 2013; Mathôt, 2018; Partala et al., 2000). In our study, we looked at the difference in pupil size averaged across three segments of $1.2 \mathrm{~s}$ within a period of 3.6 s. We found an effect of sound valence on pupillary responses, especially at an early onset and during the peak dilation $(0.4-2.8 \mathrm{~s}$ since sound onset). The effect of the sound valence was still significant but seemed to start to taper off after $2.8 \mathrm{~s}$. Interestingly, while some earlier literature reported similar pupil-size effects for negative and positive stimuli, both being larger than for neutral stimuli, we observed a different result pattern. We found that pupil size in the negative valence condition was remarkably larger than pupil size in the positive valence condition. Relative to pupil size in the neutral valence condition, there was evidence that pupil size in the negative valence condition was larger in the first two time-windows. There was also a difference in pupil size between the neutral and positive valence conditions. We found no 
Baseline-corrected

pupil size (a.u.)

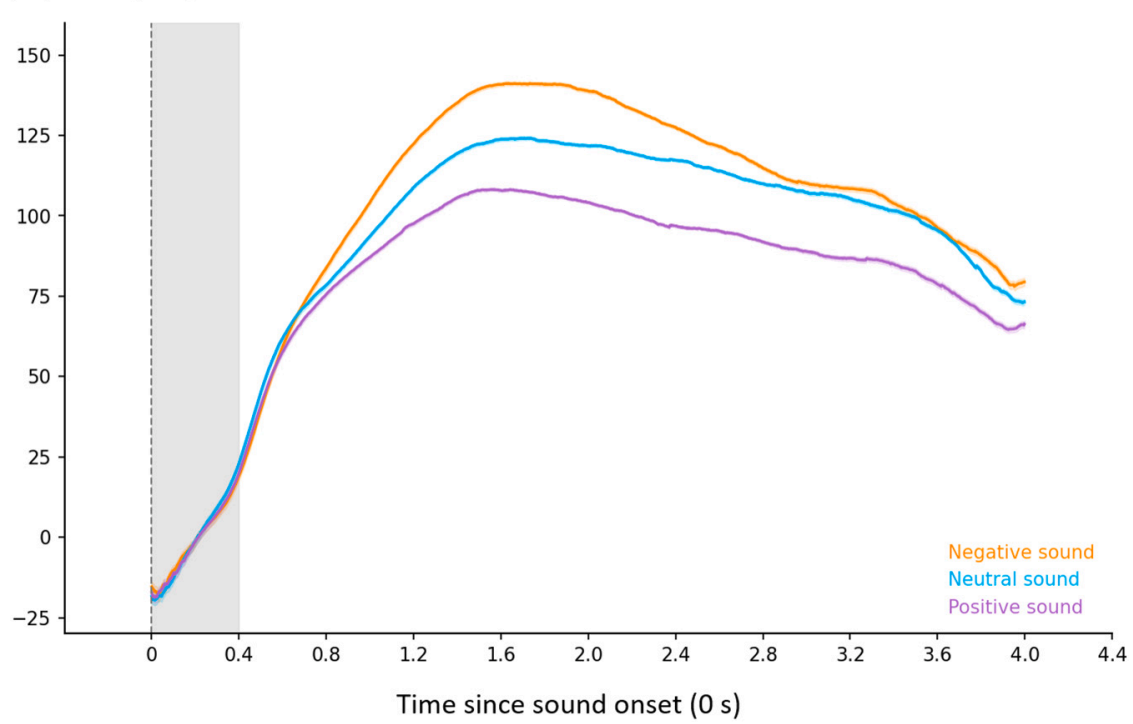

Fig. 4. Pupillary responses to sound valence.

Note. Top: The effect of sound valence explored in baselinecorrected pupil dilations, averaging over all luminance levels. Bottom: The FDR-corrected $p$-values (adjusted with Holm method; $p=.05$ as a significance threshold) presented significant effects of valence on pupil dilations in all timewindows. The pupil-dilation difference was most notable between the Negative and Positive valence. (Coloured shades around the pupil trace, although very narrow, represent $95 \% \mathrm{CI}$. The vertical grey shaded area represents a baseline period.)

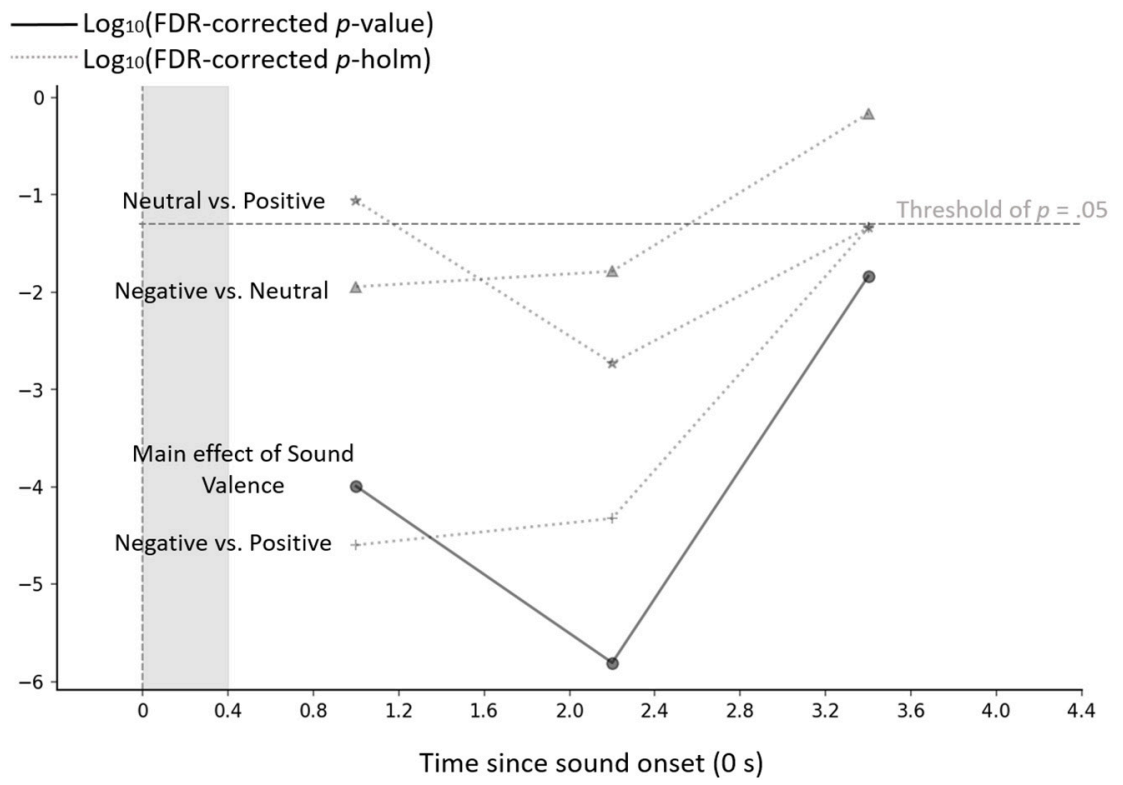

interaction between sound valence and background luminance. Taken together, these findings demonstrated that pupil dilations responding to negative, neutral, and positive sounds were not the same.

In the emotion and pupillometry literature thus far, there have been inconsistencies in the findings of the effect of emotional stimuli on pupil dilations. Most studies have shown that pupil-size changes largely reflect arousal but not a negative-positive valence difference (Bradley et al., 2008; Bradley \& Lang, 2000; Janisse, 1974). However, in our current study we have found that the difference in pupil-size changes is most notable when we compare negative and positive valence conditions, in which pupil size is the smallest in the positive valence and the largest in the negative valence condition. Importantly, this result pattern persists even after we have further equated a small residual difference in arousal rating between the different valence categories. We speculate that pupils in the positive valence condition are the smallest because the presented positive sounds are (perceived as) calming. This would be in line also with the fact that we did not select the most extreme samples on the valence dimension (i.e., not extremely negative or positive). Pupil dilation (mydriasis) and constriction (miosis) are caused by the innervations of the dilator and sphincter pupillae muscles, respectively (McDougal \& Gamlin, 2008, 2015). The autonomic nervous system contributes to this mechanism by controlling the mydriasis and miosis through the sympathetic and parasympathetic pathways, respectively. With our present finding, it is plausible that the positive sounds activate the parasympathetic pathway and participants feel rested, thus innervating the sphincter pupillae muscles that lead to smaller pupil size. In contrast, the negative sounds evoke the observers' alertness and activate the sympathetic pathway, hence larger pupil size. This supposition corroborates the findings of Cherng et al. (2020) demonstrating that emotion-induced pupillary dilations are mediated by the sympathetic activation, not parasympathetic inhibition. More surprisingly, but possibly still related to the above reasoning, neutral valence elicits larger pupil sizes than positive, which clearly contrasts with earlier work. As stated in Janisse (1974), a stimulus rated as neutral could indicate that the stimulus is ambivalent to the observer because there is a conflict or uncertainty to decide whether it is negative or positive. This proposition could explain the conflicting feeling to decide between positive and negative may increase the observers' alertness, thus innervating the 
Baseline-corrected

pupil size (a.u.)

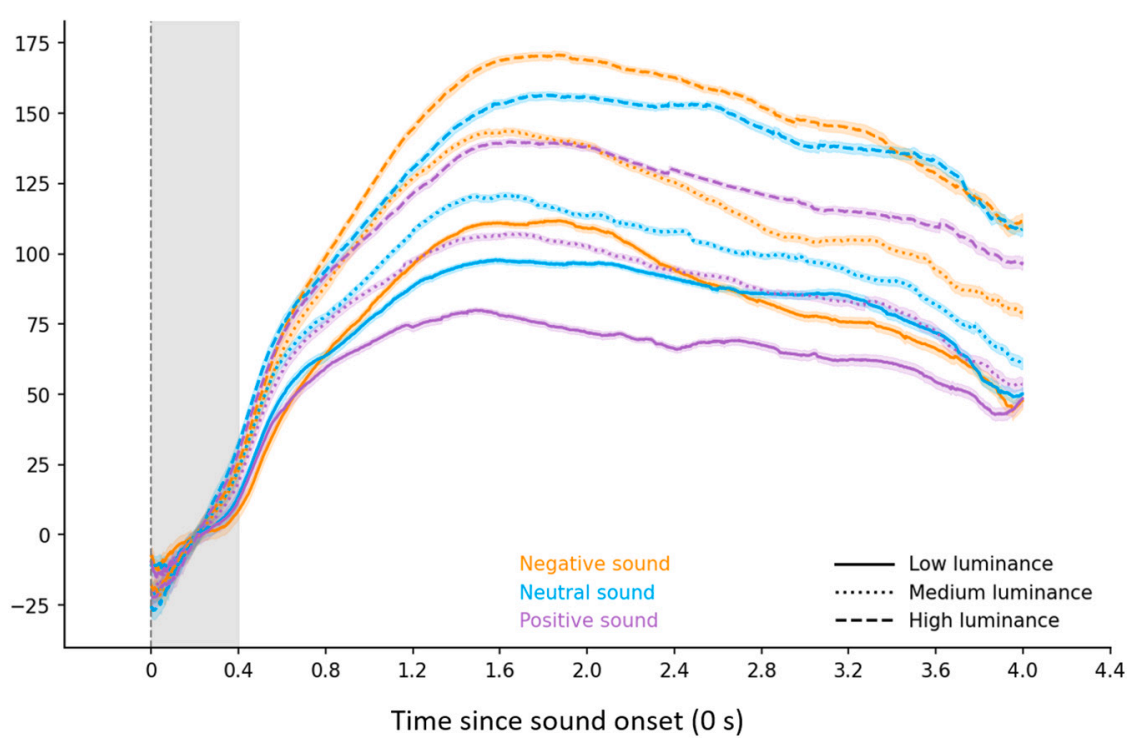

Fig. 5. Pupillary responses as a function of luminance and sound.

Note. Baseline-corrected pupil dilations as a function of background luminance and sound valence. The analysis showed that there was no significance for the interactive effect on pupil dilations. (Coloured shades around the pupil trace, although very narrow, represent $95 \%$ CI. The vertical grey shaded area represents a baseline period.) dilator pupillae muscle via the sympathetic pathway in the neutral condition.

As indicated above, we selected the sound samples to be quite well matched for arousal and dominance. For arousal, we further corroborated with an additional analysis that the observed results for valence were not confounded by arousal, which would have been a plausible alternative explanation given the link between arousal in general and pupil size (Bradley et al., 2008; Mathôt, 2018). While also trying to keep differences for the dominance dimension minimal, this did not fully succeed, which could also speak to a general non-orthogonality of the two factors in realistic sound samples. As such, our results could also be influenced by dominance, the relationship of which to pupil size has not been systematically explored yet, which future work could endeavour.

A general question that arises from this research domain is whether pupil-size adjustments through affective sounds have a functional role, in particular because pupil size can influence detection and discrimination performance (Campbell \& Gregory, 1960; Mathôt \& Ivanov, 2019; Woodhouse, 1975). The large pupil size in the negative condition may help with increasing visual sensitivity, hence detecting faint stimuli in the environment, which could be survival-relevant. In this framework, also the clear distinction to positive stimuli would make sense. Here, the small pupil size in the positive condition may serve in improving visual acuity, hence discriminating detailed stimuli, rather than having to scan the environment for possible danger. This function is useful because a discrimination task requires a relatively calm and rested state, which occurs during a positive stimulus input. Turning back to the luminance manipulation and its effect on valence processing in the present work, one could also speculate about the level at which the luminance effect on emotional processing observed here arises, and whether it converges with the functional role of sensory tuning including through peripheral mechanisms (e.g., pupil size) in perception. Given an increasing appreciation that pupil-size adjustments can serve to modulate perception (Mathôt, 2020), such a relatively direct perception-based effect also on the emotional appraisal of affective stimuli seems possible in principle. Yet, earlier work in this direction has focused on a relatively direct effect of pupil size on visual perception, for which basic optical characteristics provide a possible explanation. Given that here we investigated effects on auditory stimuli, such a mechanism would need to be multisensory in nature, and hence remains speculative.

\subsection{Conclusion and possible applications of the current findings}

In our current study, background luminance was manipulated by changing the luminance value of a blue hue on a computer screen while keeping the saturation value constant, which strongly modulated pupil size. On a behavioural level, we observed evidence for the effect of background luminance on valence ratings when presented with emotional sounds. We also inspected the possible effect of the valence of sounds on pupillary responses. Our result showed an interesting pattern in which pupil size in the positive valence condition was significantly smaller, in particular, than pupil size in the negative valence condition. Contrary to previous findings, our current finding revealed that pupillary responses might reflect not only arousal but also valence per se.

Finally, our results may be applicable in the clinical treatment for stress-related and emotion-regulation disorders. Several recent pieces of work have shown that pupil-size changes are a promising marker for post-traumatic stress symptoms and stress-related resilience (e.g., Cascardi et al., 2015; Grueschow et al., 2021; Maier \& Grueschow, 2021; Rubin \& Telch, 2021). Our pupillary results further confirm that pupil size could be a useful implicit measure of non-verbal valence perception in clinical patients. Moreover, our behavioural finding provides a new idea that could be integrated into the treatment of patients with stressrelated problems, in particular post-traumatic stress disorder (PTSD). For example, the negative response to aversive or trauma-triggering sounds could potentially be mitigated if presented in a room with enhanced blue illumination.

Supplementary data to this article can be found online at https://doi. org/10.1016/j.actpsy.2022.103532.

\section{Funding source}

The present work was supported by funding from the Flemish Research Foundation (FWO; grant no. 25 G034918N).

\section{CRediT authorship contribution statement}

I. K. Wardhani: Conceptualisation, methodology, software, formal analysis, resources, data curation, writing - review \& editing, visualisation, project administration.

B. H. Janssen: Conceptualisation, software, validation, investigation, data curation, writing - original draft 
C. N. Boehler: Supervision, project administration, funding acquisition, writing - review \& editing

\section{Declaration of competing interest}

All authors declare no conflict of interest.

\section{References}

Alkozei, A., Dailey, N. S., Bajaj, S., Vanuk, J. R., Raikes, A. C., \& Killgore, W. D. S. (2021). Exposure to blue wavelength light is associated with increases in bidirectional amygdala-DLPFC connectivity at rest. Frontiers in Neurology, 12, Article 625443. https://doi.org/10.3389/fneur.2021.625443

Benjamini, Y., \& Hochberg, Y. (1995). Controlling the false discovery rate: A practical and powerful approach to multiple testing. Journal of the Royal Statistical Society: Series B (Methodological), 57(1), 289-300. https://doi.org/10.1111/j.25176161.1995.tb02031.x

Berson, D. M., Dunn, F. A., \& Takao, M. (2002). Phototransduction by retinal ganglion cells that set the circadian clock. Science, 295(5557), 1070-1073. https://doi.org/ $10.1126 /$ science.1067262

Bradley, M. M., \& Lang, P. J. (1994). Measuring emotion: The self-assessment manikin and the semantic differential. Journal of Behavior Therapy and Experimental Psychiatry, 25(1), 49-59. https://doi.org/10.1016/0005-7916(94)90063-9

Bradley, M. M., \& Lang, P. J. (1999). International affective digitized sounds (IADS-1): Stimuli, instruction manual, and affective ratings (Technical report B-2). University of Florida, Center for Research in Psychophysiology.

Bradley, M. M., \& Lang, P. J. (2000). Affective reactions to acoustic stimuli. Psychophysiology, 37(2), 204-215. https://doi.org/10.1111/1469-8986.3720204

Bradley, M. M., \& Lang, P. J. (2007). The international affective digitized sounds: Affective ratings of sounds and instruction manual (technical report B-3). University of Florida, NIMH Center for the Study of Emotion and Attention. https://csea.phhp.ufl. edu/Media.html\#midmedia.

Bradley, M. M., Miccoli, L., Escrig, M. A., \& Lang, P. J. (2008). The pupil as a measure of emotional arousal and autonomic activation. Psychophysiology, 45(4), 602-607. https://doi.org/10.1111/j.1469-8986.2008.00654.x

Campbell, F. W., \& Gregory, A. H. (1960). Effect of size of pupil on visual acuity. Nature, 187, 1121-1123. https://doi.org/10.1038/1871121c0

Cascardi, M., Armstrong, D., Chung, L., \& Paré, D. (2015). Pupil response to threat in trauma-exposed individuals with or without PTSD. Journal of Traumatic Stress, 28(4), 370-374. https://doi.org/10.1002/jts.22022

Cherng, Y.-G., Baird, T., Chen, J.-T., \& Wang, C.-A. (2020). Background luminance effects on pupil size associated with emotion and saccade preparation. Scientific Reports, 10 (1), 15718. https://doi.org/10.1038/s41598-020-72954-z

de Winter, J. C. F., Petermeijer, S. M., Kooijman, L., \& Dodou, D. (2021). Replicating five pupillometry studies of Eckhard hess. International Journal of Psychophysiology, 165, 145-205. https://doi.org/10.1016/j.ijpsycho.2021.03.003

Faul, F., Erdfelder, E., Lang, A.-G., \& Buchner, A. (2007). G*Power 3: A flexible statistical power analysis program for the social, behavioral, and biomedical sciences. Behavior Research Methods, 39(2), 175-191. https://doi.org/10.3758/BF03193146

Gooley, J. J., Ho Mien, I., St Hilaire, M. A., Yeo, S.-C., Chua, E. C.-P., van Reen, E., Hanley, C. J., Hull, J. T., Czeisler, C. A., \& Lockley, S. W. (2012). Melanopsin and rod-cone photoreceptors play different roles in mediating pupillary light responses during exposure to continuous light in humans. The Journal of Neuroscience, 32(41), 14242-14253. https://doi.org/10.1523/JNEUROSCI.1321-12.2012

Grueschow, M., Stenz, N., Thörn, H., Ehlert, U., Breckwoldt, J., Brodmann Maeder, M. Kleim, B., ... (2021). Real-world stress resilience is associated with the responsivity of the locus coeruleus. Nature Communications, 12(1), 2275. https://doi.org/ 10.1038/s41467-021-22509-1

Hess, E. H., \& Polt, J. M. (1960). Pupil size as related to interest value of visual stimuli. Science, 132(3423), 349-350. https://doi.org/10.1126/science.132.3423.349

Hess, E. H., \& Polt, J. M. (1964). Pupil size in relation to mental activity during simple problem-solving. Science, 143(3611), 1190-1192. https://doi.org/10.1126/ science.143.3611.1190

Hess, E. H. (1965). Attitude and pupil size. Scientific American, 212(4), 46-55.

Janisse, M. P. (1974). Pupil size, affect and exposure frequency. Social Behavior and Personality, 2(2), 125-146. https://doi.org/10.2224/sbp.1974.2.2.125

Team, J. A. S. P. (2020). JASP - A Fresh Way To Do Statistics ((Version 0.14.1)) [Computer software]. JASP.

Kahneman, D., \& Beatty, J. (1966). Pupil diameter and load on memory. Science, 154 (3756), 1583-1585. https://doi.org/10.1126/science.154.3756.1583

Kawai, S., Takano, H., \& Nakamura, K. (2013). Pupil Diameter Variation in Positive and Negative Emotions with Visual Stimulus. In 2013 IEEE International Conference on Systems, Man, and Cybernetics (pp. 4179-4183). https://doi.org/10.1109/ SMC. 2013.712

Killgore, W. D. S., Dailey, N. S., Raikes, A. C., Vanuk, J. R., Taylor, E., \& Alkozei, A. (2020). Blue light exposure enhances neural efficiency of the task positive network during a cognitive interference task. Neuroscience Letters, 735, Article 135242. https://doi.org/10.1016/j.neulet.2020.135242

Laeng, B., Sirois, S., \& Gredebäck, G. (2012). Pupillometry: A window to the preconscious? Perspectives on Psychological Science, 7(1), 18-27. https://doi.org/ $10.1177 / 1745691611427305$
Lakens, D., Fockenberg, D. A., Lemmens, K. P. H., Ham, J., \& Midden, C. J. H. (2013). Brightness differences influence the evaluation of affective pictures. Cognition \& Emotion, 27(7), 1225-1246. https://doi.org/10.1080/02699931.2013.781501

Lang, P. J., Bradley, M. M., \& Cuthbert, B. N. (1997). Motivated attention: Affect, activation, and action. In P. J. Lang, R. F. Simons, \& M. T. Balaban (Eds.), Attention and orienting: Sensory and motivational processes (pp. 97-135). Lawrence Erlbaum Associates Publishers.

Lang, P. J., Greenwald, M. K., Bradley, M. M., \& Hamm, A. O. (1993). Looking at pictures: Affective, facial, visceral, and behavioral reactions. Psychophysiology, 30(3), 261-273. https://doi.org/10.1111/j.1469-8986.1993.tb03352.x

Maier, S. U., \& Grueschow, M. (2021). Pupil dilation predicts individual self-regulation success across domains. Scientific Reports, 11(1), 14342. https://doi.org/10.1038/ s41598-021-93121-y

Mathôt, S., \& Ivanov, Y. (2019). The effect of pupil size and peripheral brightness on detection and discrimination performance. PeerJ, 7, Article e8220. https://doi.org/ $10.7717 /$ peerj. 8220

Mathôt, S., Schreij, D., \& Theeuwes, J. (2012). OpenSesame: An open-source, graphical experiment builder for the social sciences. Behavior Research Methods, 44(2), 314-324. https://doi.org/10.3758/s13428-011-0168-7

Mathôt, S. (2013). A simple way to reconstruct pupil size during eye blinks. Figshare. https://doi.org/10.6084/m9.figshare.688001.v1

Mathôt, S. (2018). Pupillometry: Psychology, physiology, and function. Journal of Cognition, 1(1), 16. https://doi.org/10.5334/joc.18

Mathôt, S. (2020). Tuning the senses: How the pupil shapes vision at the earliest stage. In Annual Review of Vision Science. https://doi.org/10.1146/annurev-vision-030320062352

Mathôt, S., Fabius, J., Van Heusden, E., \& Van der Stigchel, S. (2018). Safe and sensible preprocessing and baseline correctionof pupil-size data. Behavior Research Methods, 50(1), 94-106. https://doi.org/10.3758/s13428-017-1007-2

McDougal, D. H., \& Gamlin, P. D. (2008). Pupillary control pathways. In The senses: A comprehensive reference (pp. 521-536). Elsevier. https://doi.org/10.1016/B978012370880-9.00282-6.

McDougal, D. H., \& Gamlin, P. D. (2015). Autonomic control of the eye. Comprehensive Physiology, 5(1), 439-473. https://doi.org/10.1002/cphy.c140014

Nakakoga, S., Higashi, H., Muramatsu, J., Nakauchi, S., \& Minami, T. (2020). Asymmetrical characteristics of emotional responses to pictures and sounds: Evidence from pupillometry. Plos One, 15(4), Article e0230775. https://doi.org/ 10.1371/journal.pone.0230775

Noesselt, T., Tyll, S., Boehler, C. N., Budinger, E., Heinze, H.-J., \& Driver, J. (2010). Sound-induced enhancement of low-intensity vision: Multisensory influences on human sensory-specific cortices and thalamic bodies relate to perceptual enhancement of visual detection sensitivity. The Journal of Neuroscience, 30(41), 13609-13623. https://doi.org/10.1523/JNEUROSCI.4524-09.2010

Oliva, M., \& Anikin, A. (2018). Pupil dilation reflects the time course of emotion recognition in human vocalizations. Scientific Reports, $8(1)$, 4871. https://doi.org/ 10.1038/s41598-018-23265-x

Partala, T., Jokiniemi, M., \& Surakka, V. (2000). Pupillary responses to emotionally provocative stimuli. In Proceedings of the Symposium on Eye Tracking Research \& Applications - ETRA 'OO (pp. 123-129).

Partala, T., \& Surakka, V. (2003). Pupil size variation as an indication of affective processing. International Journal of Human-Computer Studies, 59(1-2), 185-198. https://doi.org/10.1016/S1071-5819(03)00017-X

Redondo, J., Fraga, I., Padrón, I., \& Piñeiro, A. (2008). Affective ratings of sound stimuli. Behavior Research Methods, 40(3), 784-790. https://doi.org/10.3758/brm.40.3.784

Rubin, M., \& Telch, M. J. (2021). Pupillary response to affective voices: Physiological responsivity and posttraumatic stress disorder. Journal of Traumatic Stress, 34(1), 182-189. https://doi.org/10.1002/jts.22574

Sánchez-Navarro, J. P., Martínez-Selva, J. M., Román, F., \& Ginesa, T. (2006). The effect of content and physical properties of affective pictures on emotional responses. The Spanish Journal of Psychology, 9(2), 145-153. https://doi.org/10.1017/ s1138741600006041

Soares, A. P., Pinheiro, A. P., Costa, A., Frade, C. S., Comesaña, M., \& Pureza, R. (2013). Affective auditory stimuli: Adaptation of the international affective digitized sounds (IADS-2) for European Portuguese. Behavior Research Methods, 45(4), 1168-1181. https://doi.org/10.3758/s13428-012-0310-1

Spence, C. (2007). Audiovisual multisensory integration. Acoustical Science and Technology, 28(2), 61-70. https://doi.org/10.1250/ast.28.61

Stein, B. E., London, N., Wilkinson, L. K., \& Price, D. D. (1996). Enhancement of perceived visual intensity by auditory stimuli: A psychophysical analysis. Journal of Cognitive Neuroscience, 8(6), 497-506. https://doi.org/10.1162/jocn.1996.8.6.497

Stein, B. E., \& Stanford, T. R. (2008). Multisensory integration: Current issues from the perspective of the single neuron. Nature Reviews. Neuroscience, 9(4), 255-266. https://doi.org/10.1038/nrn2331

Suk, H.-J., \& Irtel, H. (2010). Emotional response to color across media. Color Research \& Application, 35(1), 64-77. https://doi.org/10.1002/col.20554

Talsma, D., Senkowski, D., Soto-Faraco, S., \& Woldorff, M. G. (2010). The multifaceted interplay between attention and multisensory integration. Trends in Cognitive Sciences, 14(9), 400-410. https://doi.org/10.1016/j.tics.2010.06.008

Thompson, R. F., Voss, J. F., \& Brogden, W. J. (1958). Effect of brightness of simultaneous visual stimulation on absolute auditory sensitivity. Journal of Experimental Psychology, 55(1), 45-50. https://doi.org/10.1037/h0047709

Valdez, P., \& Mehrabian, A. (1994). Effects of color on emotions. Journal of Experimental Psychology: General, 123(4), 394-409. https://doi.org/10.1037/00963445.123.4.394

Vallat, R. (2018). Pingouin: Statistics in python. The Journal of Open Source Software, 3 (31), 1026. https://doi.org/10.21105/joss.01026 
Vandewalle, G., Schmidt, C., Albouy, G., Sterpenich, V., Darsaud, A., Rauchs, G., Berken, P.-Y., Balteau, E., Degueldre, C., Luxen, A., Maquet, P., \& Dijk, D.-J. (2007). Brain responses to violet, blue, and green monochromatic light exposures in humans: Prominent role of blue light and the brainstem. Plos One, 2(11), Article e1247. https://doi.org/10.1371/journal.pone.0001247

van der Wel, P., \& van Steenbergen, H. (2018). Pupil dilation as an index of effort in cognitive control tasks: A review. Psychonomic Bulletin \& Review, 25(6), 2005-2015. https://doi.org/10.3758/s13423-018-1432-y

van Rij, J., Hendriks, P., van Rijn, H., Baayen, R. H., \& Wood, S. N. (2019). Analyzing the time course of pupillometric data. Trends in Hearing, 23, Article 2331216519832483. https://doi.org/10.1177/2331216519832483
Wilms, L., \& Oberfeld, D. (2018). Color and emotion: Effects of hue, saturation, and brightness. Psychological Research, 82(5), 896-914. https://doi.org/10.1007/ s00426-017-0880-8

Woodhouse, J. M. (1975). The effect of pupil size on grating detection at various contrast levels. Vision Research, 15(6), 645-648. https://doi.org/10.1016/0042-6989(75) 90278-3

Yang, W., Makita, K., Nakao, T., Kanayama, N., Machizawa, M. G., Sasaoka, T., Sugata, A., Kobayashi, R., Hiramoto, R., Yamawaki, S., Iwanaga, M., \& Miyatani, M. (2018). Affective auditory stimulus database: An expanded version of the international affective digitized sounds (IADS-E). Behavior Research Methods, 50(4), 1415-1429. https://doi.org/10.3758/s13428-018-1027-6 